\title{
Educación permanente de personas jóvenes y adultas. La línea de base como herramienta para la sistematización y producción de información cuanticualitativa*
}

\section{Lifelong education of young and adult people. The base line like tool for the systematizing and production of information cuanticualitativa}

\author{
Horacio Ademar Ferreyra** \\ Georgia Blanas de Marengo***
}

Fecha de recepción: 10 de marzo de 2011

Fecha de revisión: 27 de abril de 2011

Fecha de aprobación: 20 de mayo de 2011

* Artículo de investigación científica y tecnológica presentado por los autores en el Congreso lberoamericano de Educación. Metas 2021. Organizado por la OEI / 13, 14 y 15 de septiembre de 2010. Buenos Aires.

** Doctor en Educación de la Universidad Católica de Córdoba, Argentina, Posdoctorado en Ciencias Sociales del Centro de Estudios Avanzados de la Universidad Nacional de Córdoba, Argentina, y Posdoctorado en Ciencias Sociales de la Universidad Autónoma Metropolitana de México. Actualmente se desempeña como docente e investigador en la Facultad de Educación de la UC Córdoba, Argentina y Facultad de Educación de la Universidad Santo Tomás de Bogotá, Colombia; también es subsecretario de Promoción de Igualdad y Calidad Educativa en el Ministerio de Educación de la Provincia de Córdoba, Argentina. Ha publicado libros, ha sido coautor, ha escrito artículos en revistas científicas sobre su especialidad y participado en diversos Congresos e instancias de formación de docentes en el ámbito nacional e internacional. Correo electrónico: hferreyra@coopmorteros.com.ar

*** Doctora en Ciencia Política de la Universidad Nacional de Córdoba; asesora y consultora externa en el ámbito provincial y nacional, Córdoba, Argentina. Ha sido docente universitaria y publicado libros, artículos en revistas científicas y participado en diferentes congresos internacionales y nacionales. Correo electrónico: georgiablanas@hotmail.com 


\section{Resumen}

En Argentina la educación de personas jóvenes y adultas - EPJA, forma parte del sistema educativo, las transformaciones realizadas para ampliar la cobertura, mejorar y diversificar la oferta y adecuar los dispositivos curriculares a las cambiantes necesidades de las personas y la sociedad, no han alcanzado aún los objetivos previstos. En la producción de documentos referidos a la EPJA, se observa un déficit de información que afecta la toma de decisiones de política educativa que dificultan la optimización de la gestión del conocimiento en lo que respecta al subsistema.

Además, se desarrolla la construcción de una herramienta metodológica que permita superar los desajustes existentes ${ }^{1}$, como lo constituye la construcción de una línea de base con información sistematizada referida a la situación actual de la EPJA en Argentina, la cual permitirá promover transformaciones compatibles con los objetivos de la educación permanente dirigida a generar una educación de calidad con equidad para todos y entre todos.

Palabras clave: política, planificación y gestión educativa, línea de base, educación permanente.

\section{Abstract}

In Argentina, the education of young and adult people (ALE) is part of the educational system, the changes made to expand coverage, improve and diversify the curriculum and to adapt the devices to the changing needs of individuals and society, have not yet achieved the foreseen objectives. In the production of documents relating to the ALE, there is lack of information that affects decision-making on educational policies making it difficult to optimize the management of knowledge in regard to the subsystem.

The construction of a methodological tool to overcome the imbalance is indevelopment, as is the construction of a baseline with systematized information relating to the current situation of ALE in Argentina, which will promote changes consistent with the objectives of lifelong learning focused to generating a quality education with equity for all.

Keywords: policy, planning and educational management, baseline, lifelong education.

1 Desajustes que son consecuencia del agotamiento del modelo reformista de los noventa, que no ha sido capaz de conciliar el crecimiento cuantitativo con niveles satisfactorios de calidad y equidad. 


\section{Introducción}

En Argentina la EPJA forma parte del sistema educativo como una modalidad; si bien se han realizado y se gestionan importantes acciones para ampliar la cobertura, afianzar las relaciones interinstitucionales, mejorar y diversificar la oferta y adecuar los dispositivos curriculares a las cambiantes necesidades de las personas y la sociedad, la EPJA no alcanzó aún los objetivos previstos, en especial el de generar una educación de calidad con equidad para todos y entre todos (Consejo Provincial de Educación - CPE, 2001).

Este estado de la situación es uno de los datos más fuertes de una problemática: la educación como derecho humano universal constituye una declaración no cumplida. Ante este panorama, cabe interrogarse respecto de las posibilidades que se ofrecen, así como sobre las acciones y las políticas que se consideran deseables para romper ese círculo vicioso, comenzar a revertir la desigualdad y avanzar en un proceso de democratización educativa y social.

Por consiguiente, los indicadores son sólo una fotografía sin contexto, e impiden por sí avanzar en categorías más inclusivas de análisis, interpretación, evaluación, sobre qué tipo de educación se está ofreciendo y cuál es su vinculación con el contexto económico y social, una opción es la línea de base. Ella constituye un dispositivo práctico para el aprovechamiento y uso de la información, mientras se establece un "puente" entre la producción de información y el ejercicio de la toma de decisiones.
Es posible advertir que las preguntas, problemas y necesidades cotidianas pueden tener una respuesta mejor fundamentada y más reflexiva si se consulta la información que las distintas escalas del sistema generan en forma permanente. Así, señalamos la conveniencia de arraigarse a una cultura de recolección de datos orientada a elaborar información relevante y pertinente para la toma de decisiones.

En este contexto nos propusimos efectuar un estudio descriptivo de base cuanti-cualitativa sobre la EPJA en Argentina y, de manera particular, en las provincias de Buenos Aires, Córdoba, Mendoza, Santa Fe y Tucumán.

Es necesario mejorar los procesos y resultados en el marco de la educación, para que una educación de calidad para todos deje de ser sólo una entelequia sustentada en axiomas y se convierta, de modo efectivo, en una alternativa para la promoción de la persona humana.

\section{Marco teórico}

\section{La educación como derecho humano fundamental}

Después de algunas décadas de democracia en la región, el derecho humano a la educación sigue siendo una demanda por constituirse en la plataforma para la construcción de una nueva ciudadanía y de modelos inclusivos de sociedad. El desarrollo de estas posibilidades depende, en gran medida, de un diseño educativo estratégico situacional. 
En este sentido, la educación como "práctica social de justicia" (Derrida, 1993: 34) deberá procurar que todas las personas accedan a un conjunto de saberes y formas culturales, por medio de los cuales se favorezcan, amplíen y desarrollen sus posibilidades, a fin de permitirles comunicarse, trabajar y participar con plenitud en la sociedad, a través del ejercicio de los derechos y deberes humanos que les asisten (Ferreyra y Blanas, 2009: 43-44). Así como antes contribuyera al resurgimiento democrático, hoy el sistema educativo tiene como desafío contribuir a su transformación cualitativa.

\section{La calidad de la educación para todos}

Si bien los países que componen la región han realizado numerosos esfuerzos (aumentar los años de la educación obligatoria, incrementar la cobertura, mejorar la infraestructura, diseñar nuevos currículos y formar a los docentes, entre otros aspectos), estos no han logrado superar los problemas referidos al logro de mejorar la calidad de la educación, y esto afecta en mayor medida a las personas más vulnerables, que no se encuentran en igualdad de condiciones en lo que se refiere al acceso, la continuidad de estudios y los resultados de aprendizaje (UNESCO, 2007a, 2007b; Ministerio de Educación y del Ministerio de Trabajo, Empleo y Seguridad Social de la Nación - MTEySS, 2008).

Es este sentido, mejorar los diseños de las políticas públicas en educación y desarrollar acciones específicas serán aspectos claves si se desea lograr una educación de calidad con equidad para todos. La calidad es semejante a una espiral ascendente: una vez que se comienza a transitar su camino, alcanzar una meta es sólo un hito que supone, necesaria e inevitablemente, plantearse otra y así, sucesivamente, lograr el mejoramiento continuo.

La calidad de la educación, en tanto derecho fundamental de todas las personas, ha de reunir las siguientes dimensiones: pertinencia, relevancia, eficacia, impacto, suficiencia, eficiencia y equidad. La educación como derecho humano y bien público permite a las personas ejercer los otros derechos humanos. Asimismo, el derecho a la educación se ejerce en la medida en que las personas, más allá de tener acceso a la escuela, puedan desarrollarse plenamente y continuar aprendiendo. Esto significa que la educación ha de ser de calidad para todos y a lo largo de la vida. Por esta razón, nadie puede quedar excluido de ella.

\section{Educación permanente ${ }^{2}-E P$}

La EP abarca la vida entera de la persona. No se trata ni de un sistema, ni de un sector educativo, sino del principio en el cual se funda la organización global de un sistema y, por tanto, la elaboración de cada uno de sus subsistemas (Trilla, 1998: 51). Al respecto, Coombs (1968) afirmaba que la educación formal, y su correlato institucional, la escuela, no constituye el único ámbito en el que se manifiesta lo educativo, sino que existen también la educación no formal y la educación informal, y son estos tres secto-

2 Este apartado se ha elaborado sobre la base de los aportes de las cátedras de Educación no formal (Sentana, Cl. y. Verde, Ma. F.) y Educación de Adultos (Ferreyra, H.). Licenciatura en Ciencias de la Educación - Universidad Católica de Córdoba. Córdoba, 2009. 
res los que conforman el "universo educativo" (Sarramona, 1992: 16).

Asimismo, adherimos a la postura que sostiene que el universo educativo se encuentra íntimamente ligado al principio de educación permanente. Pero es necesario advertir que la mera existencia de tal universo no garantiza por sí misma la educación permanente, aunque sí a la inversa, ya que ésta se concreta en la medida en que involucra la totalidad de los escenarios y ámbitos educativos por los que cada sujeto transita a lo largo de su vida ${ }^{3}$.

\section{Educación permanente de jóvenes y adultos}

Desde hace más de veinte años, afirma Brusilovsky (2006: 9), distintos especialistas admiten la dificultad para adoptar una definición que aglutine las múltiples experiencias, prácticas y discursos asociados a la educación de jóvenes y adultos. Coinciden en señalar que es posible encontrar, en esta denominación, acciones que apuntan a satisfacer las aspiraciones de las personas para mejorar la condición de vida, "continuidad"; dotar de los conocimientos y de las competencias elementales (alfabetización y formación básica), "esencialidad"; lo extraescolar (no formal e informal), "libertad estructural"; la formación para el trabajo y la vida, "utilidad"; una formación en función del contexto y reali-

3 Según Trilla, la educación permanente “... abarca la biografía entera de la persona (...) y no se trata ni de un sistema, ni de un sector educativo, sino del principio en el cual se funda la organización global de un sistema y, por tanto, la elaboración de cada una de sus (las) partes que componen el universo educativo" (1998: 51). dad social, "libertad individual y de grupo", entre otras (Requejo, 2003).

En síntesis, sobre el término EPJA se maneja un conjunto de procesos de aprendizaje, formales o no, mediante los cuales las personas desarrollan sus capacidades, enriquecen sus conocimientos y mejoran sus competencias básicas y fundamentales, como así también las técnicas y profesionales, o las reorientan con el fin de atender a sus propias necesidades y a las de la sociedad (Hamburgo, 1997; Belem, 2009) De esta manera, la EPJA (formal y no formal) ocupa un espacio importante del universo educativo.

\section{Línea de base}

La línea de base marca el punto de partida y permite disponer de un patrón de análisis, comparación y progresión de los aspectos o cuestiones a trabajar desde el plan, programa o proyecto (Cañete, 1998; Ros, 2003; ME. Córdoba, 2003; MTEySS, 2008). La línea de base está indudablemente asociada a procesos de evaluación y, por tanto, a procesos de cambio, transformación, innovación y mejora (Casassus y Arancibia, 1997). Por un lado, posibilita constatar el grado de cumplimiento de los logros alcanzados en relación con las metas u objetivos propuestos. En otro sentido, permite que la información que se produce sirva de retroalimentación para constituirse en un nuevo insumo o en una nueva indagación en el proceso de cambio continuo en el que todos están involucrados (ME. Córdoba, 2003). 


\section{Diseño metodológico}

\section{Objetivo}

Construir una línea de base con información confiable del subsistema de EPJA de Argentina en general y de las provincias seleccionadas en particular con el propósito de caracterizar la situación de la EPJA en los aspectos vinculados a las instituciones, estudiantes, docentes y currículum; identificar los problemas y temas críticos que se deberían considerar en el diseño y gestión de un plan de desarrollo socioeducativo para la modalidad, cuya finalidad es la de incluir y mejorar los procesos y resultados en el marco de la educación permanente.

\section{Tipo de estudio}

Descriptivo (cuantitativo y cualitativo).

\section{Población y muestra}

La población objeto de este estudio es el subsistema de EPJA (primaria y secundaria), de la cual hemos seleccionado como muestra a las provincias de Buenos Aires, Córdoba, Santa Fe, Mendoza y Tucumán, por ser las que concentran el mayor número de instituciones educativas y población estudiantil (más del $50 \%$ del país) (DINIECE, 2006). Estas han realizado innovaciones en los últimos años, 2006-2008, por tener acceso a las fuentes de información y estadística.

\section{Procedimiento}

Sobre la base del problema de investigación y los objetivos planteados, desde el punto de vista procedimental, optamos por realizar una revisión y análisis de estudios, estadísticas y documentos producidos, durante el período comprendido entre 2006 y 2008, por distintas agencias de gestión del conocimiento. Los mismos fueron seleccionados de manera intencional por su actualidad, pertinencia y relevancia ${ }^{4}$. Además, se desarrollaron entrevistas abiertas no estructuradas a informantes claves del Ministerio de Educación y del Ministerio de Trabajo, Empleo y Seguridad Social de la Nación (6) y se realizaron observaciones no participantes en reuniones técnico-políticas con directivos provinciales y responsables de la EPJA de distintas jurisdicciones.

\section{Resultados y discusiones}

En este contexto pretendemos inscribir el texto central de nuestro trabajo, y por ello presentamos una línea de base para la EPJA en Argentina y para las provincias seleccionadas.

\section{Instituciones educativas}

Del total de 5837 instituciones educativas, en el país, al 2006, el 94,1\% es gestionado por el sector estatal. La magnitud de la participación de este sector de gestión pone en evidencia la responsabilidad que asume el Estado con aquellos que no han podido completar los niveles de enseñanza aludidos en las edades correspondientes, y la escasa participación del sector privado en la configuración de la modalidad (v. tabla 1 en el anexo).

4 Los documentos primarios consultados se encuentran destacados $\left({ }^{*}\right)$ en la referencias. 
En total, las provincias seleccionadas para este estudio concentran el $61,10 \%$ de las instituciones existentes en el país. En las provincias de Buenos Aires, Mendoza y Tucumán, el número de instituciones que ofrecen educación secundaria duplica a las de primer nivel. En cambio, en Córdoba y Santa Fe prevalecen los servicios de educación básica de nivel primario.

En cuanto a la duración de los estudios, por lo general es de seis años (tres para nivel primario y tres para nivel secundario). También se observan, en las jurisdicciones, subestructuras, por ejemplo: de cuatro años (primaria - secundaria básica), de dos años (secundaria - superior) y de tres años para cada nivel, con diferencias en la organización institucional de acuerdo con la realidad de cada jurisdicción (MECyT, 2006; Ferreyra, 2009).

Estas instituciones, en la mayoría de los casos, funcionan en horarios nocturnos o vespertinos, y casi siempre en contra turno de otros servicios escolares $u$ organizaciones intermedias, en edificios que son en la mayoría de los casos "cedidos" para la modalidad (Rodríguez, 2008; MTEySS, 2008; Ferreyra, 2009). El estado de la infraestructura y del equipamiento, en general, es precario.

Es de señalar que uno de los problemas detectados es el de la falta de condiciones adecuadas en cuanto a equipamiento e infraestructura, que repercute de modo negativo en las respectivas prácticas educativas (Ferreyra y Blanas, 2009).
Según las observaciones sobre política educativa de Luque (2008), la ausencia de proyectos sobre la problemática de diversidad e igualdad social, pone en evidencia el hecho de que muchas instituciones se resisten al cambio en cuanto a la promoción de la justicia y la equidad.

\section{Estudiantes}

\section{Matrícula}

La matrícula de EPJA totaliza en el país 629.413 estudiantes al 2006, el 23,70\% cursa primario y el $76,30 \%$ asiste al nivel secundario. En general, la EPJA se ha evidenciado un notable incremento de matrícula en la última década (1996-2006) que acumula un 89\% (DINIECE, 2006).

Las provincias seleccionadas para este estudio concentran el $61,95 \%$ (62\%) de los estudiantes matriculados en educación de jóvenes y adultos, de los que más del $40 \%$ del total del país corresponden a la provincia de Buenos Aires (v. tabla 2 en anexo). Al considerar la matrícula total, la participación por sexo registra mínimas diferencias: poco más de dos puntos a favor de las mujeres. Analizada esa dimensión por nivel educativo, se observa una caída en la participación de los varones en la educación primaria (44\%).

Si estudiamos la relación entre el total de estudiantes matriculados en primario y la cantidad de instituciones educativas de este nivel escolar en el país, podemos determinar que la proporción de estudiantes matriculados 
por institución asciende a 61. En cambio, en la educación secundaria la relación es de 141 estudiantes por IE.

Al analizar la participación de las matrículas jurisdiccionales con relación a la del país, observamos que el 40,75\% corresponde a la matrícula de provincia de Buenos Aires, y la suma de las matrículas de las demás provincias comprendidas en el presente trabajo alcanza el $21,20 \%$ del total.

En este escenario, las provincias han desplegado diversas estrategias para avanzar en el abordaje de la oferta de EDJA, al atender los nuevos requerimientos contextuales, y las particularidades de los estudiantes del sector, quienes transitan procesos de exclusión debido a múltiples causales, repetir nivel o curso, abandono, desocupación, etc. (Ministerio de Ciencia y Tecnología -MECyT, 2006; Kit, 2007; MTEySS, 2008; Ferreyra, 2009; ME, 2011).

Participación relativa por edad sobre el total de estudiantes del país por nivel de enseñanza

La participación por edad de los estudiantes de educación de jóvenes y adultos nos indica la cantidad de estudiantes por tramos de edad inscriptos en los distintos niveles de enseñanza.

Según la información estadística disponible, más del $60 \%$ de los estudiantes que asisten a los servicios de EPJA posee entre 15 y 24 años. Ellos están en edades que oscilan entre 15 y 19 años. Esto nos indica una amplia participación de adolescentes y jóvenes ${ }^{5}$.

Si analizamos la participación por nivel de enseñanza, podemos constatar que en la educación primaria la mayoría corresponde al grupo de 15 y 19 años, aunque también se destaca, en segundo lugar, la participación de personas cuyas edades oscilan entre 30 y 49 años.

Por el contrario, en la secundaria, el grupo mayoritario es el de 15 a 24 años. Allí se reconoce una participación significativa de estudiantes entre los 30 y 49 años de edad.

En el mismo sentido, observamos que el 42,3\% de los estudiantes que continúan estudios en los servicios de educación de jóvenes y adultos son menores de 20 años, lo que nos permite inferir un proceso de reinserción de jóvenes que, habiendo abandonado la educación común, se reincorporan al sistema en busca de nuevas oportunidades para completar su escolaridad (DINIECE, 2006).

Del análisis realizado se deduce que es importante reconocer e identificar cuáles son las condiciones socioeconómicas y culturales básicas para que los jóvenes y adultos puedan permanecer en el sistema educativo y efectivamente aprender, atendiendo a su singularidad.

En aquellos establecimientos en los que las condiciones de educabilidad (López y Tedesco, 2004) no están garantizadas, la escuela tiene

5 No podemos dejar de reconocer, con preocupación, la inclusión en la EPJA de niños menores de 14 años en la educación primaria (v. tabla 3 en anexo). 
un papel en su construcción. No pensamos la educabilidad como algo dado, sino como una construcción delimitada por condiciones sociales, políticas, económicas y culturales que el Estado, la comunidad y la escuela aportan a través del establecimiento de las alianzas intersectoriales necesarias (Ferreyra, 2009).

\section{Eficiencia interna en la educación de jóvenes y} adultos

Al analizar la participación de las matrículas jurisdiccionales en relación con la del país, observamos que el $40,75 \%$ corresponde a la matrícula de provincia de Buenos Aires, y la suma de las matrículas de las demás provincias comprendidas en el presente trabajo alcanza el $21,20 \%$ del total.

El análisis de la eficiencia interna permite identificar en qué medida la población matriculada en las instituciones educativas alcanza, año tras año, la acreditación correspondiente, hasta concluir con la graduación final. Una adecuada eficiencia interna se manifestaría, en primer lugar, por un alto nivel de promoción anual, cantidad de estudiantes que llegan al final del ciclo lectivo y completan los requisitos que les permite matricularse en el curso el año siguiente.

Que el estudiante no promueva o abandone los estudios es considerado un indicador de "fracaso escolar". A los efectos de este estudio, proponemos trabajar con la idea de "fracaso total" en tanto integra a quienes abandonan y no promueven, en relación con la matrícula inicial.
Al analizar los indicadores de fracaso en la $\mathrm{EPJA}^{6}$, en las provincias seleccionadas, en ambos niveles observamos que, del total de casos analizados, casi una cuarta parte de los inscriptos abandona durante el año; de quienes llegan al fin del ciclo lectivo, más de la mitad no promueve la primaria y la tercera parte la secundaria. Esto significa que, de una u otra, la mitad de la matrícula inicial de EPJA fracasa cada año interrumpiendo el proceso de escolarización, situación que propende a desocultar las causales de este fracaso, que no siempre están en el estudiantado, instituyendo la necesidad de revisar la gestión pedagógica de dichos procesos escolares (v. tablas 4 y 5 en el anexo).

Al profundizar el análisis del fracaso por niveles educativos, observamos que en el nivel correspondiente a la educación primaria se registra un mayor índice de no promoción, que alcanza casi el $54 \%$ de la matrícula final, y el abandono está próximo al $22 \%$. En la educación secundaria, el abandono se ubica en un $22 \%$, mientras que la no promoción alcanza el 33\%.

Al analizar la participación de las matrículas jurisdiccionales en relación con la del país, observamos que el 40,75\% corresponde a la matrícula de provincia de Buenos Aires, y la suma de las matrículas de las demás provincias, comprendidas en el presente trabajo, alcanza el $21,20 \%$ del total.

6 Se presenta flujo de matrícula sólo para las provincias seleccionadas en virtud de que no cuentan con información desagregada en el ámbito federal. La información presentada ha sido suministrada por las provincias seleccionadas. 
En general, nos encontramos con una modalidad que en la última década creció en cuanto a cobertura, pero que no ha logrado mejores estándares de calidad y equidad (MTEySS, 2008; Ferreyra y Blanas, 2009). Como ya se ha mencionado, las provincias han generado diversas estrategias de supervivencia con el propósito de atender los nuevos requerimientos (finalización de estudios primarios y secundarios) que fueron emergiendo en virtud de la realidad socioeconómica imperante, cuya principal manifestación está en la incorporación de los jóvenes y, recientemente, de los adolescentes excluidos de la educación común por diferentes causales -repetición de nivel o curso, abandono, sobreedad, desocupación, etc.- al escenario formativo de la EPJA. A esto se suma que muchos de ellos son desocupados o subocupados, con trabajos precarios y en el campo de lo no formal (MECyT, 2006; Kit, 2007; MTEYSS, 2008; Ferreyra, 2009; ME, 2010a, 2010c).

Al analizar en forma específica el comportamiento de los indicadores de fracaso entre las provincias seleccionadas, podemos observar que se registran diferencias según el nivel educativo:

a) Primaria: las provincias de Buenos Aires y Mendoza superan la media del fracaso total. La provincia de Buenos Aires presenta el índice más alto de abandono y en el caso de Mendoza más de la mitad de los estudiantes que llegan a fin de año, fallan en las condiciones de promoción. b) Secundaria: Mendoza y Córdoba presentan la tasa más alta de fracaso escolar total. En ambos casos se constatan altas tasas de no promoción que superan el $49 \%$, mientras que en Mendoza se evidencia un $26 \%$ de estudiantes que abandonan. Buenos Aires, Santa Fe y Tucumán se sitúan por debajo de la media del grupo en lo que respecta al fracaso total.

Esta situación indica la presencia de dificultades particulares en los sistemas provinciales, más en unos que en otros, para poder garantizar la trayectoria educativa de los estudiantes en cuanto al ingreso, permanencia, progreso y egreso, posiblemente por su dificultad para dar respuestas adecuadas al incremento de la demanda y a la incorporación masiva de nuevos públicos, que presentan privaciones socioculturales y económicas (cf. Paredes y Pochulu, 2005; Rodríguez, 2008; Kit, 2007; MTEySS, 2008; Ferreyra, 2009; Ferreyra y Blanas, 2009; ME, 2011).

\section{Egresados}

Se utiliza el término egresado para referirse al estudiante que ha cumplido con los requisitos de acreditación de los aprendizajes correspondientes a un nivel completo de enseñanza y obtenido la certificación correspondiente. También alcanza esta condición cuando completa sus estudios a través de exámenes libres y de reválida, tomando como año de egreso la fecha del último examen aprobado (DINIECE, 2006).

La salida de los estudiantes es el hito que permite la superación de la categoría del déficit escolar. Por esta razón se considera relevante 
conocer en términos absolutos y relativos el perfil del egresado en la educación secundaria.

Al respecto, cabe señalar que la distribución de los egresados en la educación primaria y secundaria por sexo también es favorable a las mujeres. En cada uno de los puntos del itinerario escolar, los indicadores expresan una mejor situación relativa de las mujeres: en matrícula, en promoción y en egreso (cf. Kit, 2007).

El porcentaje de egreso en ambos niveles como indicador dentro de la educación de jóvenes y adultos en Argentina expresa un grave problema (cf. Kit, 2007; Rodríguez, 2008; MTEySS, 2008; Ferreyra, 2009).

Si queremos dimensionar esta cantidad de egresados en relación con la necesidad global, cabe destacar que los 47.117 egresados de la educación secundaria del 2006 representan apenas el 1,96\% de los 2,4 millones de personas de 20 a 49 años que en el 2001 no habían terminado la secundaria (Kit, 2007; MTEySS, 2008) $)^{7}$.

\section{Docentes}

En la modalidad de EPJA, los docentes designados por cargos pueden cumplir tareas de dirección y gestión, frente al aula, apoyo o bien desempeñarse como personal único (DNIECE,

7 Respecto del nivel primario, no se cuenta con información desagregada por grado o curso de estudios en la educación de jóvenes y adultos en el ámbito nacional, lo que no nos ha permitido construir el dato de egresados de nivel primario. La información presentada incluye, para las provincias de Buenos Aires, Mendoza y Tucumán, los egresados del ciclo básico de la educación secundaria. v. tabla 6 en el anexo.
2006), sin tener en cuenta si acreditan formación para esos ámbitos determinados ${ }^{8}$.

De la totalidad de cargos asignados a la modalidad, el 70,86\% corresponde al nivel secundario. Si consideramos ambos niveles, predominan los cargos frente a estudiantes: el $73 \%$. Se destaca también la cantidad de personal de apoyo asignado en nivel medio: 5458 docentes, que corresponde al $13,7 \%$ de los cargos de la modalidad en el país.

Si analizamos la relación entre el total de cargos docentes en primario y la matrícula total del nivel en el país, podemos observar que la proporción es de un cargo docente cada 14 estudiantes. En cambio, en la educación secundaria la proporción es de 17 estudiantes.

Cabe señalar que si tomamos sólo los cargos frente a estudiantes, la relación en primaria asciende a 18 estudiantes por cargo y en secundaria a 23 estudiantes por cargo.

Por otro lado, cabe destacar que la mayoría del personal directivo y docente no ha sido formado, en principio, para trabajar en la modalidad. Por lo tanto, son sus designaciones a término fijo o interinas lo que dificulta la tarea en cuanto a su especificidad y a su continuidad para poder brindar una educación de calidad entre adultos (MECyT, 2006; Rodríguez, 2008; MTEySS, 2008; Ferreyra, 2009).

8 Para los fines del cálculo estadístico en el presente trabajo, las horas cátedra han sido convertidas a cargo: 20 horas cátedra equivalen a 1 cargo y los mismos se han sumado a las funciones frente a aula. v. tabla 7 en el anexo, ahí presentamos la información estadística disponible. 
No obstante, se destacan algunas experiencias de formación en servicio a través de carreras de postítulo o posgrado, a las que pueden acceder los profesores de educación primaria o EGB 1 y 2 o de secundaria - media o EGB 3 y polimodal, como también cursos desarrollados en el marco de la Red Federal de Formación Docente continua a los fines de formarse en cuestiones específicas de la modalidad.

\section{Contenidos curriculares y modalidades de enseñanza}

Asumimos el currículum como un proyecto cultural, social, político y educativo que se elabora en un momento histórico determinado. Su finalidad radica en organizar experiencias de enseñanza y aprendizaje -a escala nacional, jurisdiccional y/o escolar- que posibilitan herramientas a los estudiantes para actuar críticamente en la sociedad en que viven. En nuestro caso, centramos el análisis en el ámbito nacional y de las provincias seleccionadas y en dos de sus componentes: contenidos y modalidades.

Respecto de los contenidos, no se ha producido, en el ámbito nacional, una propuesta curricular específica para la modalidad, sino que, sobre la base de los contenidos generales para todo el sistema educativo (contenidos básicos comunes) y vinculados a la acreditación de cada uno de los niveles (primario o secundario), se efectúan -en la mayoría de las jurisdicciones- adecuaciones curriculares (Ferreyra, 2009).
No obstante, en el caso de las provincias seleccionadas se han desarrollado planes, programas de estudios y diseños específicos para la modalidad. Los estudios pueden cursarse, en la mayoría de los casos, en la estrategia presencial, aunque en los últimos años se está incorporando la semipresencial y a distancia, como alternativas para garantizar oportunidades formativas a toda la población (Ferreyra, 2009). En el caso de las ofertas presenciales, la carga horaria diaria de clases varía entre dos y cuatro horas y en la semipresencialidad, la asistencia oscila de dos o tres días a la semana (MECyT, 2006).

\section{Iniciativas y experiencias significativas}

El Estado ha implementado iniciativas que procuran brindar oportunidades educativas a las personas jóvenes y adultas. El desafío educativo de cada una de las provincias se concentra en reconstruir el tejido social y político de los distintos actores responsables en sus respectivos contextos. En este sentido, se han considerado algunas experiencias vigentes en los sistemas objetos de este estudio, en términos de su potencialidad, para contribuir a las estrategias de transformación ${ }^{9}$ :

9 Para ampliar la información sobre experiencias relevantes en las provincias seleccionadas v. MTEySS. Informes de organismos provinciales y de gerencias de empleo y capacitación laboral. SE. DNOyFP, Argentina, 2007; Rodríguez, L. (2008). Situación presente de la educación de jóvenes y adultos en Argentina. México: CREFAL. recuperado el 21 de diciembre de 2008 de http://www.crefal. edu.mx/descargas/informes_nacionales/argentina.pdf y Piñón, F. (coord.) (2008). Hacia una sociedad más justa. La educación de los jóvenes y adultos en América Latina: experiencias y desafíos. Buenos Aires: Editorial Santillana. 
Experiencias significativas

\section{Buenos Aires}

- Educación a distancia (primaria y secundaria); trabajo intersectorial para la orientación y el seguimiento de los estudiantes.

- Apoyo a jóvenes en la búsqueda de trabajo y mejoramiento de las condiciones socioeconómicas de las mujeres de zonas rurales.

- Centros de orientación y apoyo para los estudiantes, para que finalicen los estudios secundarios.

- Articulación del nivel secundario a distancia con la formación profesional.

\section{Córdoba}

- Programa de alfabetización y educación básica "Nunca es tarde".

- Educación a distancia - modalidad adultos - nivel secundario.

- Centros integrados de educación de jóvenes y adultos (articulación de la formación básica y la técnica).

- Programa de alfabetización y educación básica "Nunca es tarde".

- Educación a distancia - modalidad adultos - nivel secundario.

- Centros integrados de educación de jóvenes y adultos (articulación de la formación básica y la técnica).

\section{Mendoza}

- Itinerarios formativos articulados IFA, de educación básica y formación profesional.

- Mendoza productiva - formación profesional.

- Capacitación docente en la construcción curricular para la modalidad presencial y semipresencial; formación docente para el régimen no graduado de educación básica para adultos.

\section{Santa Fe}

- Diseño curricular específico para el nivel primario para jóvenes y adultos.

- Capacitación docente en políticas de promoción y retención.

- Apoyo a los estudiantes con materiales didácticos y el desarrollo del módulo de proyecto ocupacional para los estudiantes.

\section{Tucumán}

- Programa de entrenamiento para el trabajo del joven tucumano.

- Servicio de orientación y de intermediación laboral.

Consideramos que las diferentes iniciativas señaladas se constituyen en referentes necesarios para el diseño e implementación de políticas de mejora para la EPJA. Las propuestas que surjan del escrutinio crítico de las prácticas socioeducativas deberán contemplar la puesta 
en marcha de acciones de seguimiento y evaluación de la calidad, que suministren información referida a los logros, dificultades e impacto social de las estrategias implementadas.

\section{Conclusión}

El estudio expuesto nos ha permitido avanzar en la búsqueda de información y la construcción de conocimiento sobre EPJA en nuestro país y las provincias seleccionadas, y reemplazar las estrategias sustentadas en el desarrollo de meros diagnósticos por la construcción de una línea de base que sistematiza información cuanticualitativa significativa y a la vez relevante.

Dicha línea nos ha permitido establecer la situación inicial del escenario EPJA, proporcionando información respecto a sus instituciones, estudiantes, docentes, contenidos curriculares, modalidad de enseñanza e iniciativas y experiencias innovadoras, con la intencionalidad de identificar tensiones, problemas y temas críticos para la definición de políticas, y constituirse en un punto de referencia para que en futuras investigaciones o evaluaciones se pueda determinar en qué grado se van alcanzando o no los objetivos propuestos.

En este sentido, se creía conveniente, una vez sistematizada la información, enunciar los problemas y temas críticos que se deberían considerar en el diseño y gestión de un plan de desarrollo socioeducativo para la modalidad.
Al avanzar en el trabajo de análisis de la información, hemos identificado los siguientes problemas situacionales, a saber:

- Incapacidad para dar respuestas adecuadas al incremento de la demanda y a la incorporación masiva de nuevos públicos (adolescentes y jóvenes) que presentan profundas diferencias etarias, sociales, culturales, educativas y económicas.

- Impedimentos para brindar, de manera integrada, una formación que posibilite a los estudiantes la construcción de sus respectivos proyectos de vida como ciudadanos.

- Dificultades para diseñar y desarrollar una propuesta curricular integrada y a la vez articulada, que responda específicamente a la EPJA ${ }^{10}$.

- Escasa capacidad de diseño e implementación de modalidades de enseñanza alternativas (semipresencial y a distancia).

- Diversificación de modelos institucionales -escuela para la educación primaria o secundaria, centros de formación profesional para la formación para el trabajo, etc.- que

10 Debido a que persiste una organización centrada en contenidos más que en el desarrollo de capacidades y/o competencias, estructurados en áreas, disciplinas con escasa o casi nula conexión entre ellas, en su mayoría bajo la modalidad presencial, en tiempos y espacios presenciales y a la vez poco flexibles; carencia de materiales educativos específicos para la educación de jóvenes y adultos; además, deterioro y falta de infraestructura y mobiliario adecuados para el desarrollo de las tareas formativas. A esto se suma la falta de reconocimiento de los saberes que, aunque no estén certificados, han sido adquiridos y desarrollados por el joven-adulto en la educación común o en la vida. 
dificultan la configuración de una propuesta formativa integral para la modalidad.

- Deficiente nivel de logro alcanzado por los estudiantes, que se manifiesta en los altos índices de abandono y repetición, en el bajo rendimiento en los exámenes de ingreso a los estudios superiores y en las dificultades para el ingreso al mundo del trabajo.

- Devaluación social de los títulos y certificaciones.

- Desajustes normativos que dificultan la operatividad del sistema.

- Régimen laboral y condiciones de trabajo docente poco apropiados, basados en un sistema de asignación de funciones por cargos u horas cátedra (interinas a término) que no permiten la construcción de la carrera como profesional de la educación de adultos, y baja calidad y escasas instancias de capacitación en servicio y de especialización.

- Dificultades para construir estrategias de articulación de la EJPA con la trama social, cultural y productiva de los contextos.

A partir de estos problemas, surge una serie de temas críticos de la agenda educativa que en la actualidad están recibiendo consideraciones diversas y dando origen a múltiples respuestas, convergentes y divergentes, como:

- Expansión, diversidad y equidad

- Funciones

- Currículum

- Modalidad de enseñanza

- Configuración institucional

- Trayectoria escolar de los estudiantes

- Títulos y certificaciones

- Normativa

- Desarrollo laboral y profesional de los docentes

- Relaciones con la comunidad

Cada uno de los temas mencionados está relacionado con los demás, de manera tal que cada uno de ellos constituye un punto de partida para ingresar en el análisis de los diferentes aspectos del problema de la EPJA, como es el de su identidad y especificidad, en el marco de la aplicación de la Ley Nacional de Educación, no sólo para las diferentes provincias seleccionadas, sino también para otras que configuran el sistema educativo nacional. 


\section{Anexo}

\begin{tabular}{|l|r|r|r|r|}
\hline \multicolumn{5}{|c|}{ Tabla 1. Instituciones educativas por nivel de enseñanza (2006) } \\
\hline Total país / provincias seleccionadas & Total & $\begin{array}{c}\text { \% participación } \\
\text { jurisdicción }\end{array}$ & \multicolumn{1}{c|}{ Primario } & Secundario \\
\hline Total país & 5837 & 100,00 & 2444 & 3393 \\
\hline Buenos Aires & 2229 & 38,00 & 679 & 1550 \\
\hline Córdoba & 447 & 7,70 & 305 & 142 \\
\hline Mendoza & 366 & 6,30 & 104 & 262 \\
\hline Santa Fe & 382 & 6,60 & 237 & 145 \\
\hline Tucumán & 143 & 2,50 & 37 & 106 \\
\hline Fuente: autor, información suministrada por DINIECE-RA, 2006. & & & \\
\hline
\end{tabular}

\begin{tabular}{|c|c|c|c|c|c|c|c|}
\hline \multirow{2}{*}{$\begin{array}{l}\text { Total país / provin- } \\
\text { cias seleccionadas }\end{array}$} & \multicolumn{2}{|l|}{ Total } & \multicolumn{2}{|c|}{ Primario } & \multicolumn{2}{|c|}{ Secundario } & \multirow{2}{*}{$\begin{array}{c}\text { \% } \\
\text { participación } \\
\text { jurisdiccional }\end{array}$} \\
\hline & Estudiantes & $\begin{array}{c}\% \\
\text { Mujeres }\end{array}$ & Estudiantes & $\begin{array}{c}\% \\
\text { Mujeres }\end{array}$ & Estudiantes & $\%$ Mujeres & \\
\hline Total país & 629.413 & 52,4 & 149.439 & 56,0 & 479.974 & 51,3 & 100 \\
\hline Buenos Aires & 256.522 & 51,9 & 37.236 & 59,9 & 219.286 & 50,6 & 40,75 \\
\hline Córdoba & 45.899 & 54,4 & 9017 & 58,2 & 36.882 & 52,4 & 7,30 \\
\hline Mendoza & 27.301 & 50,0 & 5742 & 48,3 & 21.559 & 50,5 & 4,30 \\
\hline Santa Fe & 39.618 & 56,6 & 18.642 & 58,1 & 20.976 & 55,2 & 6,30 \\
\hline Tucumán & 20.601 & 50,0 & 7001 & 48,5 & 13.600 & 50,7 & 3,25 \\
\hline
\end{tabular}

\begin{tabular}{|l|r|r|r|}
\hline \multicolumn{4}{|c|}{$\begin{array}{c}\text { Tabla 3. Participación relativa por edad sobre total de estudiantes del } \\
\text { país por nivel de enseñanza (2006) }\end{array}$} \\
\hline Participación relativa por edad & \multicolumn{1}{|c|}{ Total } & Primario & Secundario \\
\hline Estudiantes de 14 años y menos & $3,1 \%$ & 12,63 & 0,60 \\
\hline Estudiantes de 15 a 19 años & $39,2 \%$ & 32,12 & 40,64 \\
\hline Estudiantes de 20 a 24 años & $21,6 \%$ & 9,01 & 25,07 \\
\hline Estudiantes de 25 a 29 años & $9,8 \%$ & 8,00 & 11,00 \\
\hline Estudiantes de 30 a 49 años & $21,5 \%$ & 25,78 & 19,72 \\
\hline Estudiantes de 50 y más años & $4,8 \%$ & 12,46 & 2,97 \\
\hline Fuente: MECYT - RAMCs, 2006. \\
\hline
\end{tabular}


EDUCACIÓN PERMANENTE DE PERSONAS JÓVENES Y ADULTAS. LA LÍNEA DE BASE COMO HERRAMIENTA...

\begin{tabular}{|c|c|c|c|c|c|c|c|c|c|c|}
\hline $\begin{array}{c}\text { Provincias } \\
\text { seleccionadas }\end{array}$ & $\begin{array}{l}\text { Matrícu- } \\
\text { la inicial }\end{array}$ & $\begin{array}{l}\text { Abando- } \\
\text { no anual }\end{array}$ & $\begin{array}{c}\% \\
\text { Aban } \\
\text { dono }\end{array}$ & $\begin{array}{l}\text { Matrícula } \\
\text { final }\end{array}$ & Promovidos & $\begin{array}{l}\text { \% pro- } \\
\text { moción }\end{array}$ & $\begin{array}{l}\text { No pro- } \\
\text { movidos }\end{array}$ & $\begin{array}{c}\% \\
\text { no promo- } \\
\text { ción anual }\end{array}$ & $\begin{array}{l}\text { Fracaso total } \\
\text { (no promo- } \\
\text { ción más } \\
\text { abandono) }\end{array}$ & $\begin{array}{c}\% \\
\text { fracaso } \\
\text { total }\end{array}$ \\
\hline Total & 77.638 & 16.743 & 21,60 & 60.895 & 28.224 & 46,30 & 32.671 & 53,70 & 49414 & 63,60 \\
\hline Buenos Aires & 37.236 & 9719 & 26,10 & 27.517 & 12.520 & 45,50 & 14.997 & 54,50 & 24.716 & 66,40 \\
\hline Córdoba & 9017 & 974 & 10,80 & 8043 & 3716 & 46,20 & 4327 & 53,80 & 5301 & 58,80 \\
\hline Mendoza & 5742 & 1108 & 19,30 & 4634 & 1840 & 39,70 & 2794 & 60,30 & 3902 & 67,90 \\
\hline Santa Fe & 18.642 & 3486 & 18,70 & 15.156 & 7320 & 48,30 & 7836 & 51,70 & 11.322 & 60,73 \\
\hline Tucumán & 7001 & 1456 & 20,80 & 5545 & 2828 & 51,00 & 2717 & 49,00 & 4173 & 59,60 \\
\hline
\end{tabular}

\begin{tabular}{|c|r|r|r|r|r|r|r|r|r|r|}
\hline \multicolumn{2}{|c|}{ Tabla 5. Matrícula inicial, abandono, matrícula final y promovida - no promovida, educación } \\
\hline $\begin{array}{c}\text { Provincias } \\
\text { seleccionadas }\end{array}$ & $\begin{array}{c}\text { Matrícula } \\
\text { inicial }\end{array}$ & $\begin{array}{c}\text { Abando- } \\
\text { no anual }\end{array}$ & $\begin{array}{c}\text { secundaria de jóvenes } y \text { adultos (2006) } \\
\text { aban- } \\
\text { dono }\end{array}$ & $\begin{array}{c}\text { Matrícula } \\
\text { final }\end{array}$ & Promovidos & $\begin{array}{c}\% \text { pro- } \\
\text { moción }\end{array}$ & $\begin{array}{c}\text { No pro- } \\
\text { movidos }\end{array}$ & $\begin{array}{c}\text { \% promo- } \\
\text { no } \\
\text { ción anual }\end{array}$ & $\begin{array}{c}\text { Fracaso } \\
\text { total (no pro- } \\
\text { moción más } \\
\text { abandono) }\end{array}$ & $\begin{array}{c}\text { \% } \\
\text { fracaso } \\
\text { total }\end{array}$ \\
\hline Total & 312.303 & 66.482 & 21,30 & 245.821 & 165.255 & 67,20 & 80.566 & 32,80 & 147.048 & 47,10 \\
\hline Buenos Aires & 219.286 & 52.904 & 24,10 & 166.382 & 119.098 & 71,60 & 47.284 & 28,40 & 100.188 & 45,70 \\
\hline Córdoba & 36.882 & 4093 & 11,10 & 32.789 & 16.637 & 50,70 & 16.153 & 49,30 & 20.246 & 54,90 \\
\hline Mendoza & 21.559 & 5623 & 26,10 & 15.936 & 8026 & 50,40 & 7910 & 49,60 & 13.533 & 62,10 \\
\hline Santa Fe & 20.976 & 2250 & 10,70 & 18.726 & 13.339 & 71,20 & 5386 & 28,20 & 7636 & 36,40 \\
\hline Tucumán & 13.600 & 1612 & 11,90 & 11.988 & 8155 & 68,00 & 3833 & 32,00 & 5445 & 40,10 \\
\hline Fuente: autor, información suministrada por las provincias, 2006. & & & & \\
\hline
\end{tabular}

Tabla 6. Egresados del nivel primario y secundario (2006)

\begin{tabular}{|c|c|c|c|c|}
\hline \multicolumn{5}{|c|}{ Tabla 6. Egresados del nivel primario y secundario (2006) } \\
\hline \multirow{2}{*}{ Provincias seleccionadas } & \multicolumn{2}{|c|}{ Primario } & \multicolumn{2}{|c|}{ Secundario } \\
\hline & Estudiantes & $\%$ Mujeres & Estudiantes & $\%$ Mujeres \\
\hline Total país & 53.798 & 55,7 & 47.117 & 59,0 \\
\hline Buenos Aires & 24.440 & 59,8 & 21.342 & 61,1 \\
\hline Córdoba & 1542 & 58,0 & 5563 & 59,0 \\
\hline Mendoza & 1733 & 51,0 & 1512 & 64,5 \\
\hline Santa Fe & 3970 & 55,0 & 3215 & 59,8 \\
\hline Tucumán & 198 & 50,0 & 777 & 55,7 \\
\hline
\end{tabular}




\begin{tabular}{|c|c|c|c|c|c|c|c|c|c|c|}
\hline \multirow[b]{2}{*}{$\begin{array}{l}\text { División } \\
\text { político- } \\
\text { territorial }\end{array}$} & \multirow[b]{2}{*}{ Total } & \multicolumn{5}{|c|}{ Primario } & \multicolumn{4}{|c|}{ Secundario } \\
\hline & & Total & Dir y G & $\begin{array}{c}\text { Frente a } \\
\text { estudiantes }\end{array}$ & Apoyo & $\begin{array}{l}\text { Personal } \\
\text { único }\end{array}$ & Total & Dir y G & $\begin{array}{c}\text { Frente a } \\
\text { estudian- } \\
\text { tes }\end{array}$ & Apoyo \\
\hline Total país & 39.849 & 10.813 & 1501 & 8375 & 875 & 62 & 29.036 & 2835 & 20.743 & 5458 \\
\hline Buenos Aires & 15.615 & 2949 & 488 & 2295 & 165 & 1 & 12.666 & 694 & 8886 & 3086 \\
\hline Córdoba & 2425 & 467 & 11 & 405 & 39 & 12 & 1958 & 195 & 1579 & 184 \\
\hline Mendoza & 1791 & 478 & 33 & 436 & 9 & - & 1313 & 160 & 989 & 164 \\
\hline Santa Fe & 2347 & 1237 & 174 & 962 & 90 & 11 & 1110 & 319 & 750 & 41 \\
\hline Tucumán & 1461 & 671 & 62 & 447 & 162 & - & 790 & 95 & 554 & 141 \\
\hline
\end{tabular}

\section{Referencias}

Brusilovsky, S. (2006). Educación escolar de adultos. Una identidad en construcción. Buenos Aires: NOVEDUC.

Consejo Provincial de Educación (2001). Propuestas generales para los diseños curriculares. Santa Cruz, Argentina: Dirección de Adultos.

Coombs, P. (1985). La crisis mundial de la educación. Perspectivas actuales. Madrid: Editorial Santillana.

Derrida, J. (1993). Los espectros de Marx. París: Galilée.

DINIECE (2005-2007). Anuario estadístico.

Ferreyra, H. (2009). La educación permanente de personas jóvenes y adultas en la República Argentina. El caso de las provincias de Buenos Aires, Córdoba, Mendoza, Santa
Fe y Tucumán (2006-2008) [tesis posdoctoral]. México, D.F: Universidad Autónoma Metropolítina de México.

Ferreyra, H. y Blanas, G. (2009). La construcción de una línea de base en el campo de la educación de personas jóvenes y adultas en la República Argentina (2006). Córdoba, Argentina: Editorial Mimeo.

Kit, I. (2007). Evolución reciente y situación actual de la educación de adultos en Argentina 1997-2005. Educación para todos Asociación civil. Recuperado el 14 de diciembre de 2008 de http://www.chubut. edu.ar/descargas/secundaria/congreso/ ADULTOS/RLE3476_Ferreyra.pdf

López, N. y Tedesco, J.C. (2004). Condiciones de educabilidad de los niños y adolescentes en América Latina. Buenos Aires: IIPE.

Luque, M. (2008). Implicaciones de la mejora de la calidad de la educación en el desarrollo 
de los pueblos. Conferencia presentada ante la ANEP. Uruguay.

Ministerio de Educación, Ciencia y Tecnología (2006). Gestión curricular para la educación de jóvenes y adultos [documento de trabajo]. Argentina.

Ministerio de Educación, Ciencia y Tecnología (2010). Trayectoria socioeducativa de jóvenes y adultos. Investigación, núm. 1. Argentina: DINIECE (a). Recuperado el 5 de mayo del 2011 de http://diniece.me.gov. ar/images/stories/diniece/publicaciones/ serie/informe/01-serie-investigacion.pdf

Ministerio de Educación, Ciencia y Tecnología (2010). Principales ideas, discusiones y producciones EPJA. Investigación, núm. 2. Argentina: DINIECE (b).

Ministerio de Educación, Ciencia y Tecnología (2010). Aportes para pensar la educación de jóvenes y adultos en el nivel secundario. Un estudio desde la perspectiva de los sujetos. Serie Educación en Debate, núm. 7. Argentina: DINIECE (c).

Ministerio de Educación, Ciencia y Tecnología (2011). La producción de investigaciones y estudios en temas de la EDJA en Argentina. Un estudio desde la perspectiva de los sujetos. Serie Educación en Debate, núm. 8. Argentina: DINIECE.
Ministerio de Trabajo, Empleo y Seguridad Social (2007). Informes de Organismos Provinciales y de Gerencias de Empleo y Capacitación Laboral. Documento de trabajo. Argentina.

Ministerio de Trabajo, Empleo y Seguridad Social (2008). La educación como política de empleo en la Argentina. El caso de la educación de jóvenes y adultos en las provincias de Buenos Aires, Córdoba, Mendoza, Santa Fe y Tucumán. Argentina: Dirección Nacional de Orientación y Formación profesional. Subsecretaría de Políticas de Formación Profesional y Empleo MTEySS. Buenos Aires.

Paredes, S.M. y Pochulu, M.D. (2005). La institucionalización de la educación de adultos en la Argentina. Revista Iberoamericana de Educación, 36(8) - Organización de Estados Iberoamericanos para la Educación, la Ciencia y la Cultura (OEI).

Piñón, F. (Coord.) (2008). Hacia una sociedad más justa. La educación de los jóvenes y adultos en América Latina: experiencias y desafíos. Buenos Aires: Editorial Santillana.

Requejo, A. (2003). Educación permanente y educación de adultos. Barcelona: Editorial Ariel. 
Rodríguez, L. Situación presente de la educación de jóvenes y adultos en Argentina. México: CREFAL. Recuperado el 21 de diciembre de 2008 de www.crefal.edu.mx/ descargas/informes_nacionales/argentina. pdf

Trilla, J. (1998). La educación fuera de la escuela. Barcelona: Editorial Ariel.
UNESCO (2002-2007). Proyecto Regional de Educación para América Latina y El Caribe - PRELAC. Cuba-Argentina.

V Conferencia Internacional CONFINTEA (1997). Hamburgo, Alemania.

VI Conferencia Internacional CONFINTEA (2009). Belem, Brasil. 